\title{
A!
}

This is an electronic reprint of the original article.

This reprint may differ from the original in pagination and typographic detail.

Volovik, G.E.; Yakovenko, V.M.

\section{Hopf term for a two-dimensional electron gas}

Published in:

Physical Review Letters

DOI:

10.1103/PhysRevLett.79.3791

Published: 01/01/1997

Document Version

Publisher's PDF, also known as Version of record

Please cite the original version:

Volovik, G. E., \& Yakovenko, V. M. (1997). Hopf term for a two-dimensional electron gas. Physical Review Letters, 79(19), 3791. https://doi.org/10.1103/PhysRevLett.79.3791

This material is protected by copyright and other intellectual property rights, and duplication or sale of all or part of any of the repository collections is not permitted, except that material may be duplicated by you for your research use or educational purposes in electronic or print form. You must obtain permission for any other use. Electronic or print copies may not be offered, whether for sale or otherwise to anyone who is not an authorised user. 


\section{Hopf Term for a Two-Dimensional Electron Gas}

In a recent Letter [1], Apel and Bychkov presented a microscopic calculation of the prefactor $\Theta$ of the topological Hopf term in the effective action of a twodimensional electron gas in a magnetic field at the filling factor $\nu=1$. This derivation is questionable. The integrand in Eq. (14) of Ref. [1], which supposedly contains the Hopf term, is the total spatial derivative

$$
-\partial_{x}\left[\cos \bar{\theta} \frac{\partial(\bar{\phi}, \bar{\psi})}{\partial(t, y)}\right]+\partial_{y}\left[\cos \bar{\theta} \frac{\partial(\bar{\phi}, \bar{\psi})}{\partial(t, x)}\right] .
$$

The space integral of Eq. (1) vanishes, if the field $\mathbf{n}$ is rotated so that $\cos \bar{\theta}(\infty)=0$. Since the Hopf term is invariant under such rotation, this means that Eq. (14) of Ref. [1] does not contain the Hopf term at all. This zero result may be an artifact of the parametrization of the rotation matrices and the unit vector $\mathbf{n}$ by the Euler angles $\bar{\theta}, \bar{\phi}$, and $\bar{\psi}$, which is potentially dangerous, because $\bar{\phi}$ and $\bar{\psi}$ are ill defined when $\cos \bar{\theta}= \pm 1$.

We developed the proper method of calculating $\Theta$ microscopically in Ref. [2] for ${ }^{3} \mathrm{He}-\mathrm{A}$ films (see also Ref. [3]). This method was used to calculate $\Theta$ for various lattice models in Ref. [4] and for quasi-onedimensional conductors in Ref. [5]. The parametrization in terms of the Euler angles was avoided in Refs. [2-5].

The results of Ref. [4] apply to a general class of mean-field fermion models characterized by a microscopic Hamiltonian of the form

$$
\hat{H}=\hat{H}_{0}+\boldsymbol{\sigma} \cdot \mathbf{n}(\mathbf{r}, t) \hat{H}_{1} .
$$

In Hamiltonian (2), which acts on the electron wave functions, the Pauli matrices $\sigma$ act on the spin indices of the electrons, $\mathbf{n}(\mathbf{r}, t)$ is a unit vector slowly varying in the $(2+1)$-dimensional space-time, and the spinindependent Hamiltonians $\hat{H}_{0}$ and $\hat{H}_{1}$ are such that the system has an insulating energy gap at the Fermi level. (In the case of the BCS superconducting gap [2], the equations below are similar, but somewhat modified.) As shown in Refs. [2,4], the effective action of model (2) (obtained by integrating out the electrons) contains the Hopf term, whose coefficient $\Theta$ is given by the following expression (in the normalization of Ref. [1]):

$$
\Theta=\pi N
$$

where $N$ is the integer-valued topological invariant in the momentum space (see also Ref. [6])

$$
N=\frac{1}{4 \pi^{2}} \operatorname{Tr} \int d \omega d k_{x} d k_{y} G \frac{\partial G^{-1}}{\partial \omega} \frac{\partial G^{-1}}{\partial k_{x}} G \frac{\partial G^{-1}}{\partial k_{y}} .
$$

In Eq. (4), $k_{x}$ and $k_{y}$ are the electron momenta in the $x$ and $y$ directions, $\omega$ is the Wick-rotated frequency, and

$$
G\left(\omega, k_{x}, k_{y}\right)=\left[i \omega-\hat{H}_{0}\left(k_{x}, k_{y}\right)-\sigma_{z} \hat{H}_{1}\left(k_{x}, k_{y}\right)\right]^{-1}
$$

is the Green function of the electrons. [To derive the
Hopf term, we locally transform the electrons $\psi^{\prime}=$ $\hat{U}(\mathbf{r}, t) \psi$ to make $\mathbf{n}$ parallel to the $z$ axis and expand the effective action in the gradients of $U(\hat{\mathbf{r}}, t)[2,4]$.] The topological invariant (4) also determines the quantized Hall conductivity of the system:

$$
\sigma_{x y}=N e^{2} / h,
$$

so $\Theta$ and $\sigma_{x y}$ are proportional to each other.

Thus, for a model (2), a microscopic derivation of $\Theta$ amounts to substituting the Green function of the model into Eq. (4) and doing the integral. Since the meanfield Hartree-Fock model of Ref. [1] belongs to the class (2), Eqs. (3), (4), and (6) should apply to this model. Comparing the value of the Hall conductivity at $\nu=1$ with Eq. (6), one finds that $N=1$, thus, from Eq. (3), $\Theta=\pi$, as suggested in Ref. [1]. Strictly speaking, integral (4) has to be somewhat modified for this model, because $k_{x}$ and $k_{y}$ are not good quantum numbers in the magnetic field simultaneously. That amounts to replacing the integration over the momenta by averaging over the phases of the boundary conditions, which is standard in the quantum Hall effect theory [7]. Recently, Iordanskii and Plyasunov [8] derived $\Theta$ microscopically for the model of Ref. [1] following the approach of Ref. [2].

The effective action approach applies only to the spin textures of a large size. The quantum statistics of smallsize Skyrmions is discussed in Ref. [9].

$$
\begin{aligned}
& \text { G. E. Volovik }{ }^{1,2} \text { and V.M. Yakovenko }{ }^{3} \\
& { }^{1} \text { Helsinki University of Technology } \\
& \text { Low Temperature Laboratory } \\
& \text { P.O. Box 2200, FIN-02015 HUT, Finland } \\
& { }^{2} \text { Landau Institute for Theoretical Physics } \\
& 117334 \text { Moscow, Russia } \\
& { }^{3} \text { Department of Physics } \\
& \text { University of Maryland } \\
& \text { College Park, Maryland 20742-4111 }
\end{aligned}
$$

Received 26 March 1997 [S0031-9007(97)04497-9]

PACS numbers: 73.20.Dx, 71.35.Ji, 73.20.Mf, 75.30.Et

[1] W. Apel and Yu. A. Bychkov, Phys. Rev. Lett. 78, 2188 (1997).

[2] G.E. Volovik and V.M. Yakovenko, J. Phys. Condens. Matter 1, 5263 (1989).

[3] G. E. Volovik, Exotic Properties of Superfluid ${ }^{3} \mathrm{He}$ (World Scientific, Singapore, 1992).

[4] V. M. Yakovenko, Phys. Rev. Lett. 65, 251 (1990); Fizika (Zagreb) 21, 231 (1989); E-print cond-mat/9703195.

[5] V. M. Yakovenko, Phys. Rev. B 43, 11353 (1991).

[6] D. V. Khveshchenko and P.B. Wiegmann, Mod. Phys. Lett. B 3, 1383 (1989).

[7] Q. Niu, D. J. Thouless, and Y.-S. Wu, Phys. Rev. B 31, 3372 (1985).

[8] S. V. Iordanskii, Pis'ma Zh. Eksp. Teor. Fiz. 66, 178 (1997) [JETP Lett. 66, 188 (1997)]; S. V. Iordanskii and S. G. Plyasunov, E-print cond-mat/9706236.

[9] J. Dziarmaga, Phys. Rev. B 55, 10654 (1997). 